\title{
Flexible Endoscopy and Cervical Dysplasia Grade 1 and 2
}

\author{
Anter SS* \\ Obstetrics, Gynecology, Cairo University, Egypt
}

*Corresponding author: Salwa Samir anter, MD, Obstetrics, Gynecology, Cairo

University, Egypt, Tel: 01067324010, 01222654548; Email:

arsalwaantr8866@gmail.com

\section{Image Article}

Cervical intraepithelial neoplastic grads1 were studied by flexible endoscopy obtaining five images: First image obtaining using white light the second and third image obtaining by blue, green narrow band image respectively by the special technique of endoscopy the fourth image was obtaining after that acetic acid was applied lastly, the iodine stain is applied and the fifth image was recorded after end of examination the five image were recorded and reviewed to be studied in correlation with cytology and histopathology results to be obtaining accurate diagnosis for CIN1.

The resulting of the study that CIN1 lesion not accurate diagnosis by light white only. Both acetic acid and iodine stain determine only the extent of lesion but obscure underlying pathological changes whiles in blue and green band image both the vascular and the mucosa change were easy to be studied add to that the pathology of cells as met aplastic change reserve cells can be evaluated also all parts of cervix as the exocervix transformation zone and the endocervix can be studied by manual manipulation of endoscopic narrow band imaging (NBI) is an optical image enhancement technology that uses two short wavelength light beams that are $415 \mathrm{~nm}$ (blue) and $540 \mathrm{~nm}$ (green), [1]. It is an endoscopic imaging technique for the enhanced visualization of micro vascular architecture and micro surface structure between the epithelial surface and subjacent vascular pattern [2].

Flexible magnifying endoscopy is at present time used for the gastrointestinal tract and is tolerable for the diagnosis of GI neoplasms. Magnifying endoscopy with narrow band imaging can be used to clearly imagine the microstructures of the mucosal surface and interstitial capillaries as regard of used of flexible endoscopy examination cervix lesion [3]. The first was studied by Nishiyama, et al. [4] was reported the used of endoscopy for diagnosing cervical neoplasms revealed microvascular pattern differences at different stages [5]. The second study of K Uchita, et al. [6] study feature findings of high-grade cervical intraepithelial neoplastic or more on magnifying endoscopy with narrow band imaging [6].

So, it is anticipated to be useful for diagnosing CIN1. This study aimed to identify characteristic findings of CIN1 visualized using flexible endoscopy by five images and confirmed by the cytology result. Cervical al intraepithelial neoplastic grade 1 (CIN1) is a lesion of basal cells consisting in an architecture disorganization and cytological atypia limited to the lower third of the cervical epithelium. It is considered as a precancerous lesion uterine cervix carcinoma while they spontaneously regress in more than $60 \%$ of cases in two years [7-9].

The common procedure has been traditional for uterine cervical cancer is the Pap smear followed by colposcopy CIN1 site and character is distribution usually at squamous columnar junction and flood in the area of immature metaplasia but any part of transformation zone can be affected it affected squamous epithelium of exocervix even the endocervix can be affected the distribution may univocal to multifocal. CIN1 characteristic of small but multiple distinct and randomly scattered in or outside transformation zones. The contour of lesion is macular but in few cases maculopapular with irregular border and the margin is indistinct demarcation from normal tissue but it may flocculation or geographic appearance figure 1 . 


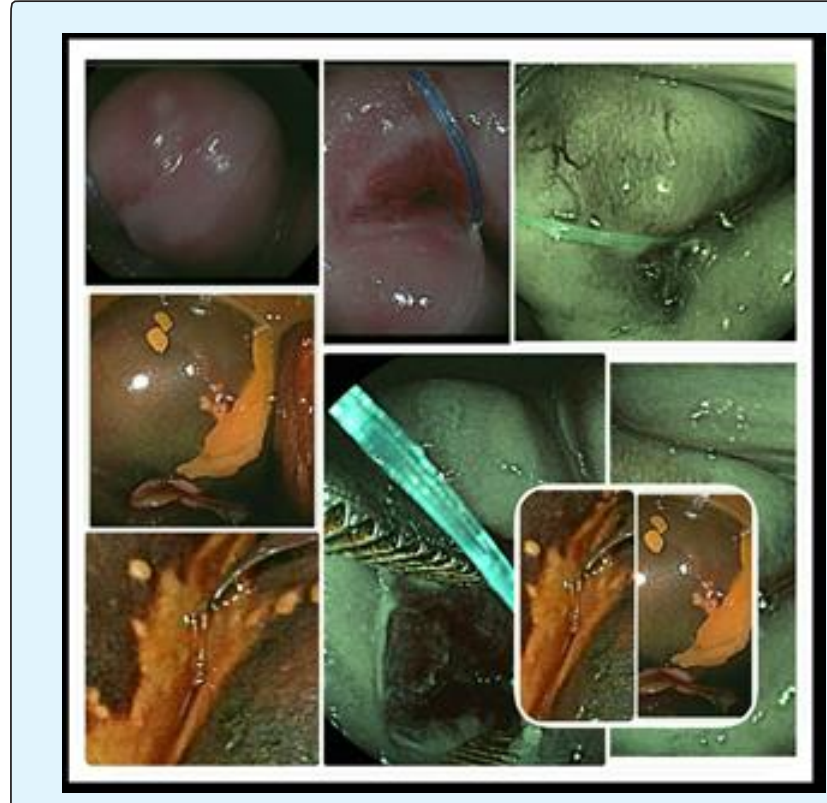

Figure 1: CIN1the site and the character in different image on the exocervix in first image, in squamous columnar junction in second image.

Color of CIN1 by white light of endoscopic is normal pink epithelium to be acetowhite on addition of acetic acid on addition of iodine CIN 1 lesions station with a mustard yellow in the area that were formerly acetowhite lesion. The image by green narrow band cervix appears green in color but the lesion area different in color and thickness appears deep green or greenish brown color of deep vessels brown elevated areas of dysplasia and nabathion cyst the color of blue narrow band the cervix appears bluish green in color while lesion area is elevated thick and more deep greenish to brown in color and the pink to brownish area represent the blood vessels and the black dots of donate the opening of cervical gland and pink to brown dots of blood vessels. Figures $2 \mathrm{a}, 2 \mathrm{~b} \& 2 \mathrm{c}$ Vessels character of CIN1.

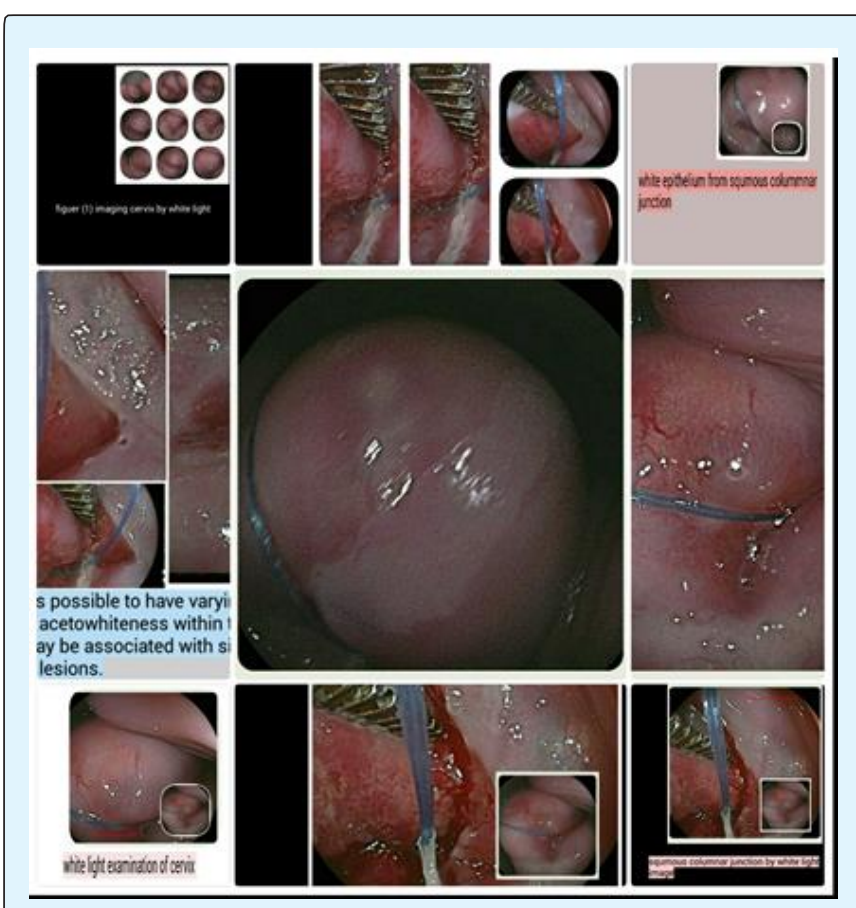

Figure 2a: Color of CIN1 before and after acetic acid application. Color of CIN1 by white light of endoscopic is normal pink epithelium to be acetowhite on addition of acetic acid.

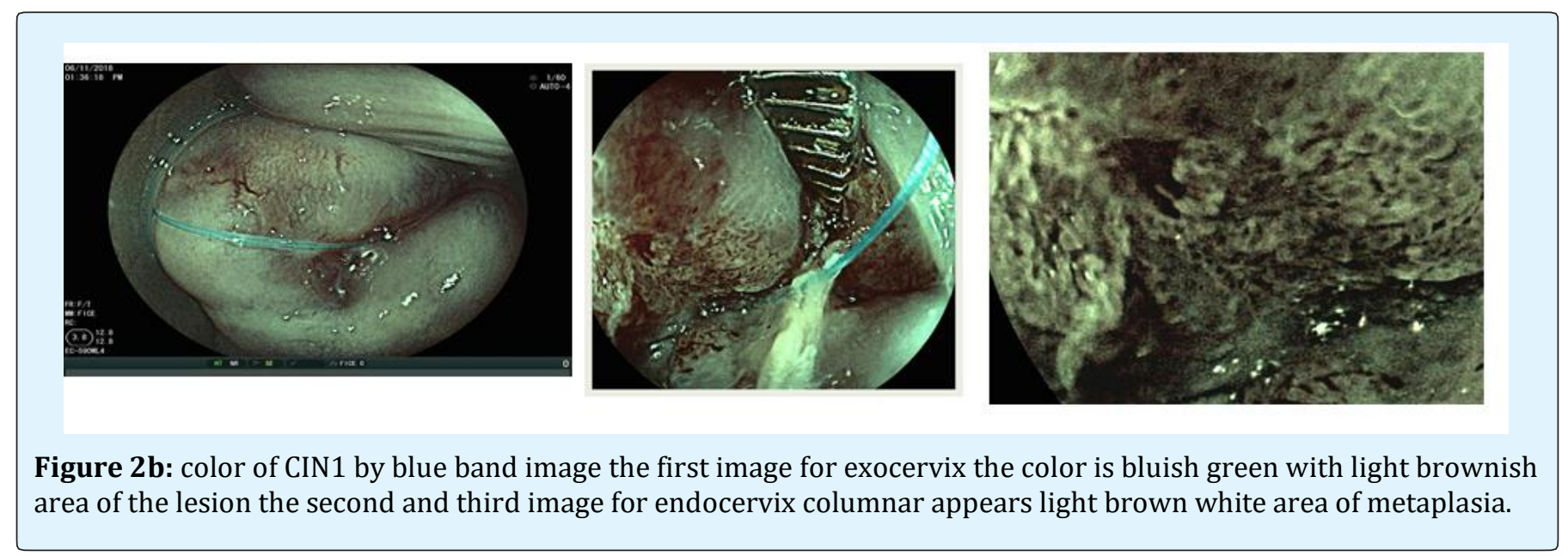




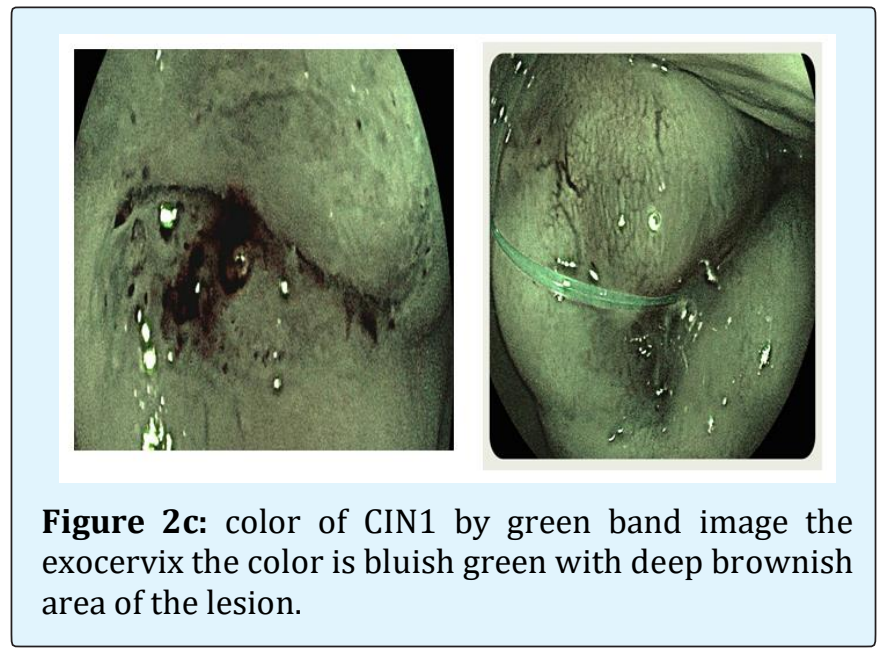

The vascular change of mild dysplasia were studied by white light image blue band image and green band image but in acetowhite area it is difficult to evaluated due to obscure of light to reach deep to vessel also no blood vessel seen in area with iodine negative endoscopic image both the micro vascular pattern and the micro surface pattern vascular pattern of blue narrow band studied the superficial capillaries and mucosa while green narrow band represent the deep vessels and the sub mucosa structure vascular character of mild dysplasia the vessels arranged uniformly have a fine, narrow lumen and the spacing between as in normal tissue vessels but the intracapillary distant is slightly increased. The resulting of dysplasia of epithelium cells on blood vessels was fine punctuation is closely uniformly equally distributed tiny dots red color in white light image but color is different it appears brown in green band image purple in Color in blue band image.

The mosaic vascular pattern is homogeneous in distribution and appears homogeneous in distribution image obvious in both blue and green band image Figure 3. Lugol's iodine stain CIN1 appears mustard yellow color not taken iodine so it is the iodine negative site is the same area of the acetowhite area And in the area of increase density of capillary and mosaics vascular and punctuation and black point and irregular surface in other area variegated yellow and brown pattern as CIN1occure in basal and parabasal layers and Glycogen is present in intermediate and superficial layers of squamous cells figures 4,4a, figure 5 CINI by blue, green and iodine figure $5 \mathrm{~b}$ CINI by blue narrow image, figure $5 \mathrm{c}$ CINI by blue narrow image. Study of transformation zone and squamous columnar junction the squamous columnar junction is the area of infection by viral so must be examined and also, the areas of metaplasia must be detected as the viral infections in which the viral engulfed but the can found in any areas of the transformation zone or in squamous epithelium on exocervix but also endocervix can be affected by viral Pagsite is important in diagnosis of CIN10 figure $6,6 a$ ion cyst by 3 image showing increased density of capillaries and thickness of mucosa lining the cyst and deep green in blue band image in areas of CIN1 figure 7.
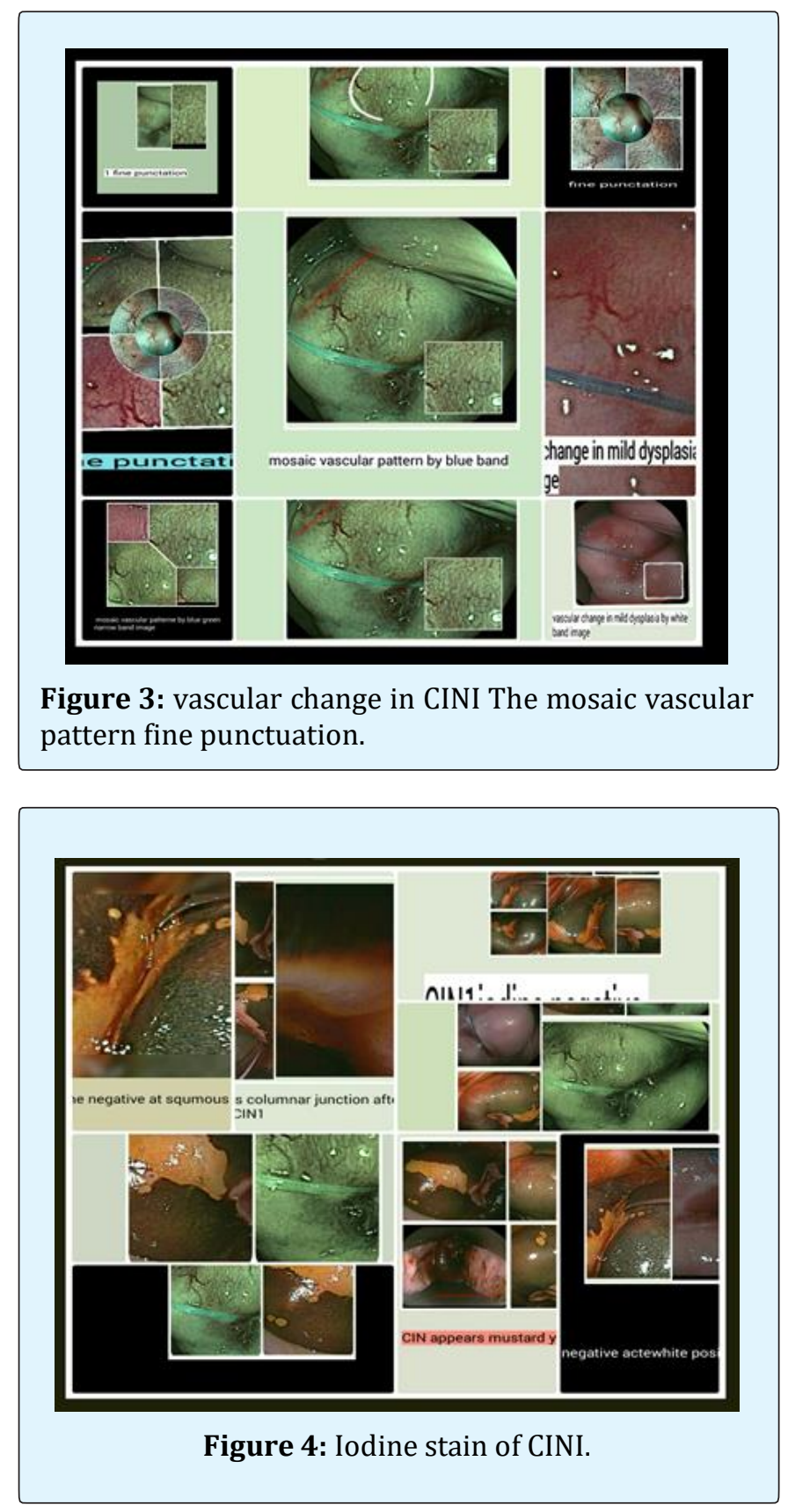

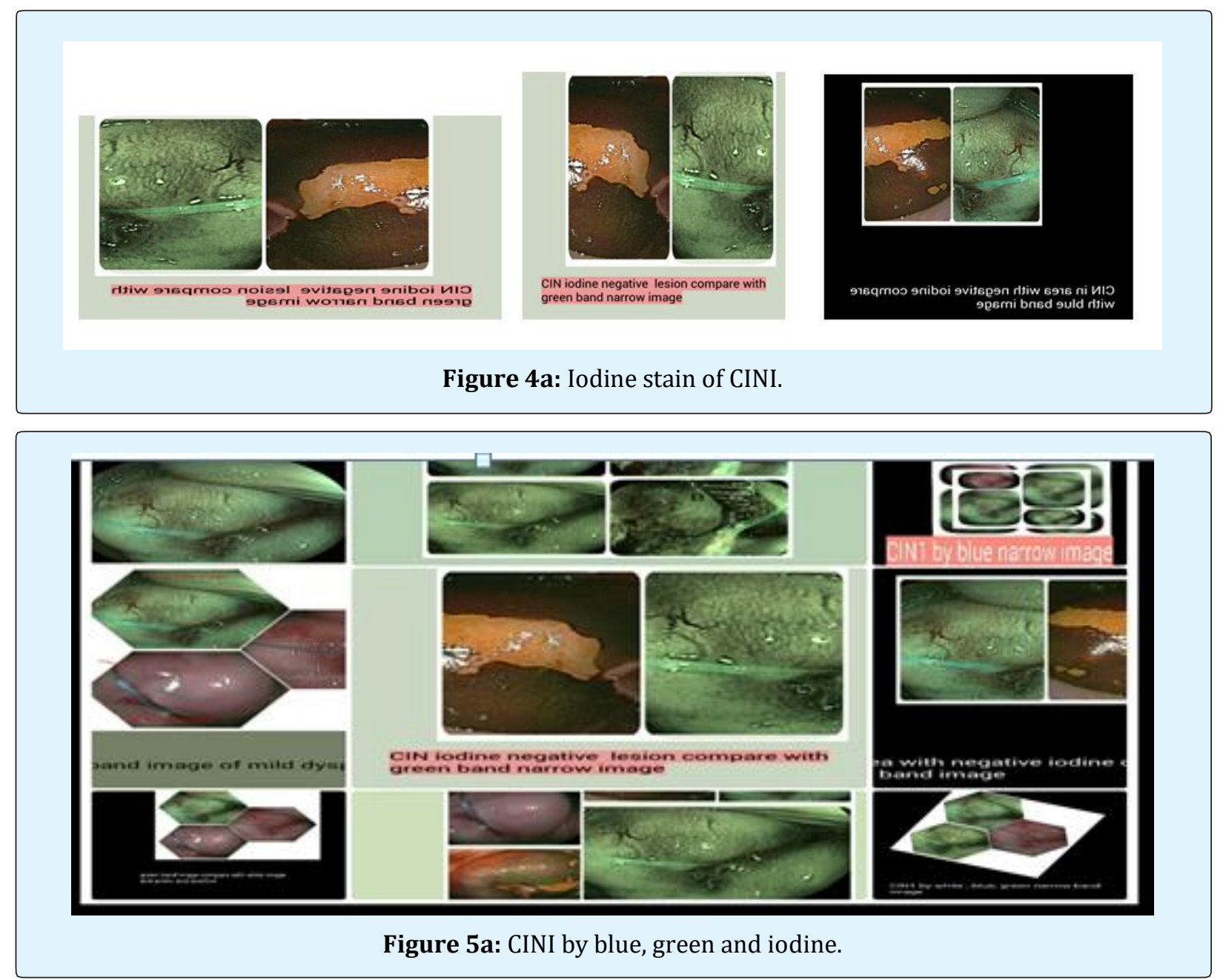

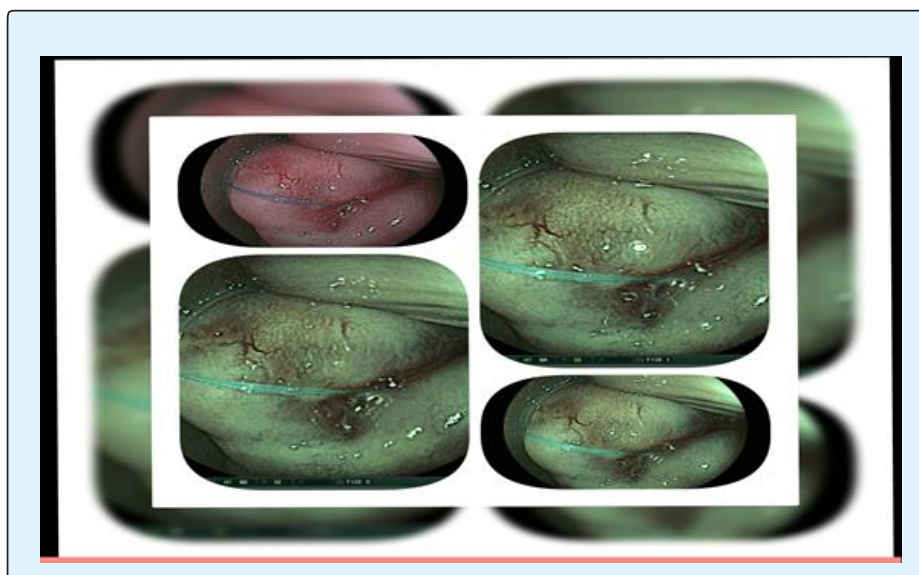

Figure 5b: CINI by blue narrow image.

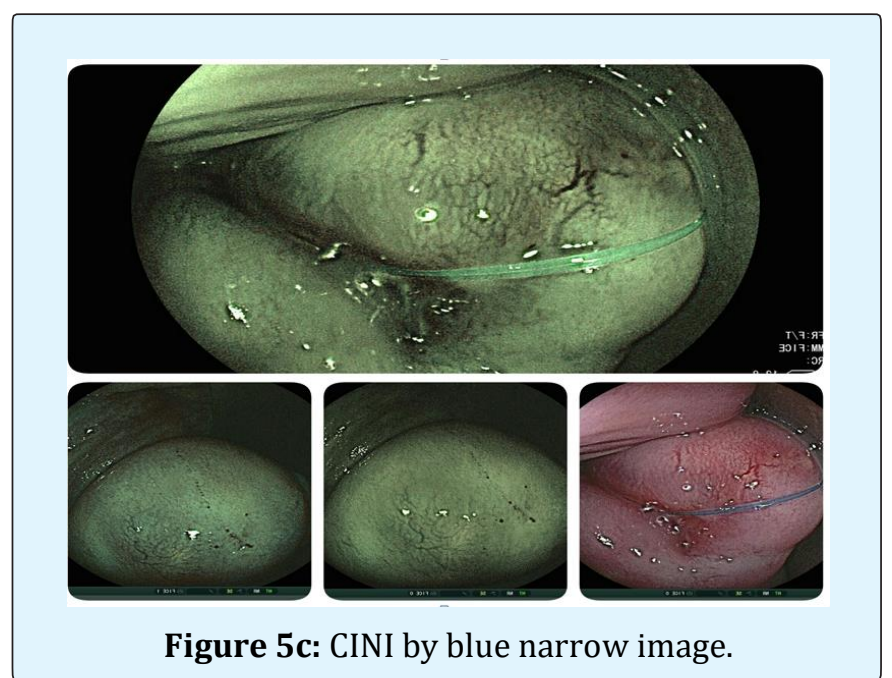

Figure 5c: CINI by blue narrow image. 


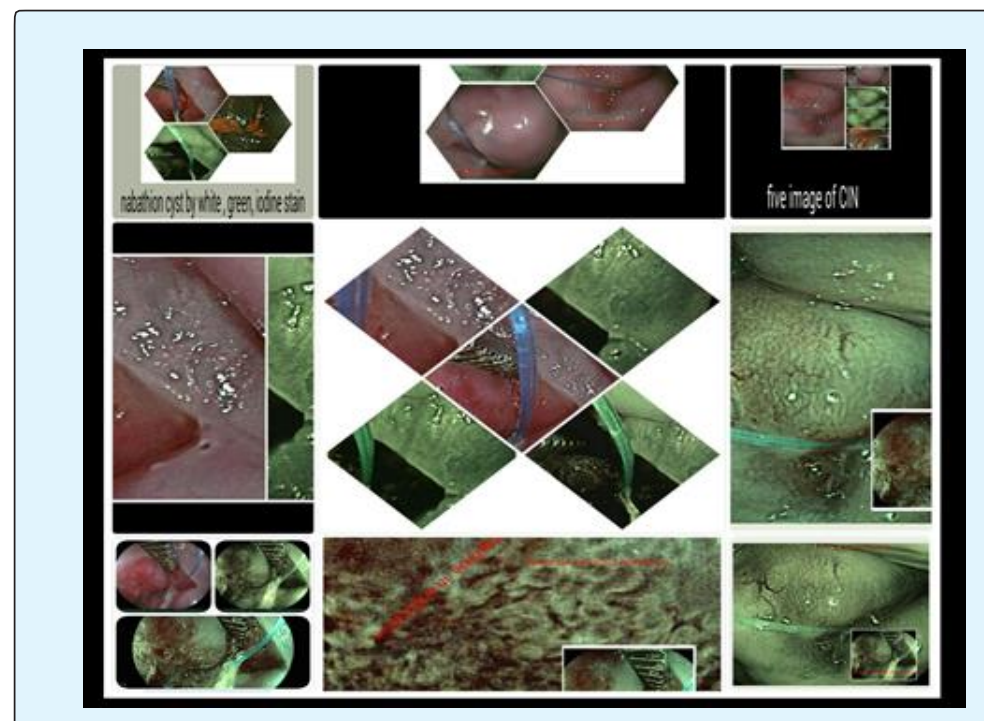

Figure 6: Squmous colummnar junction.

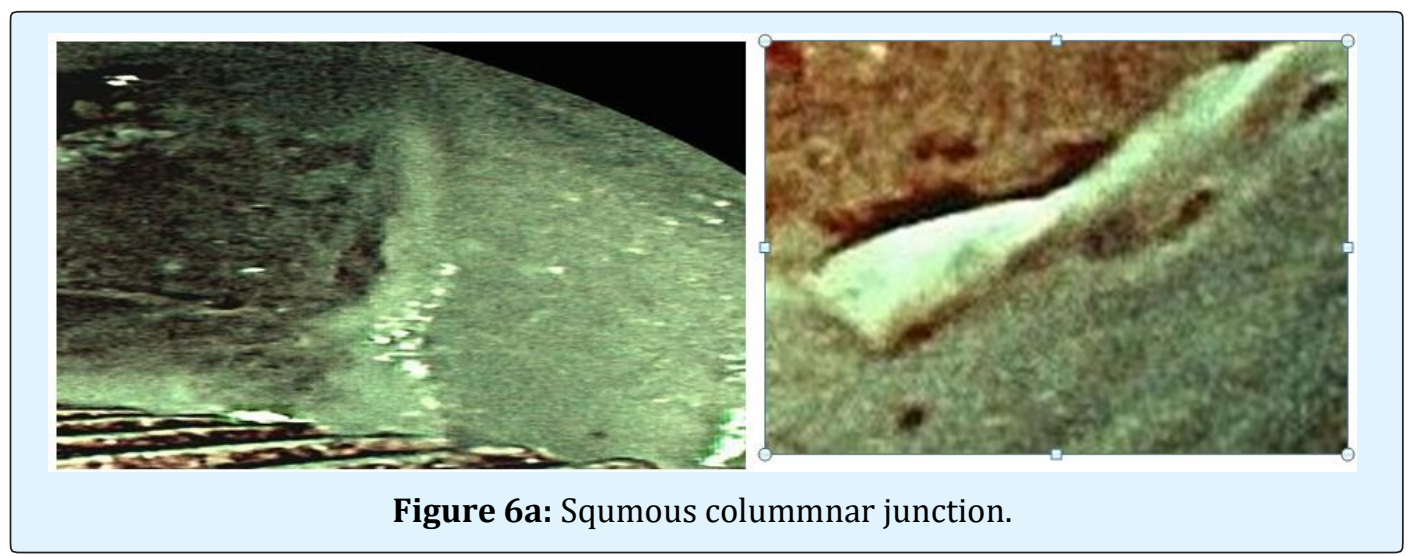

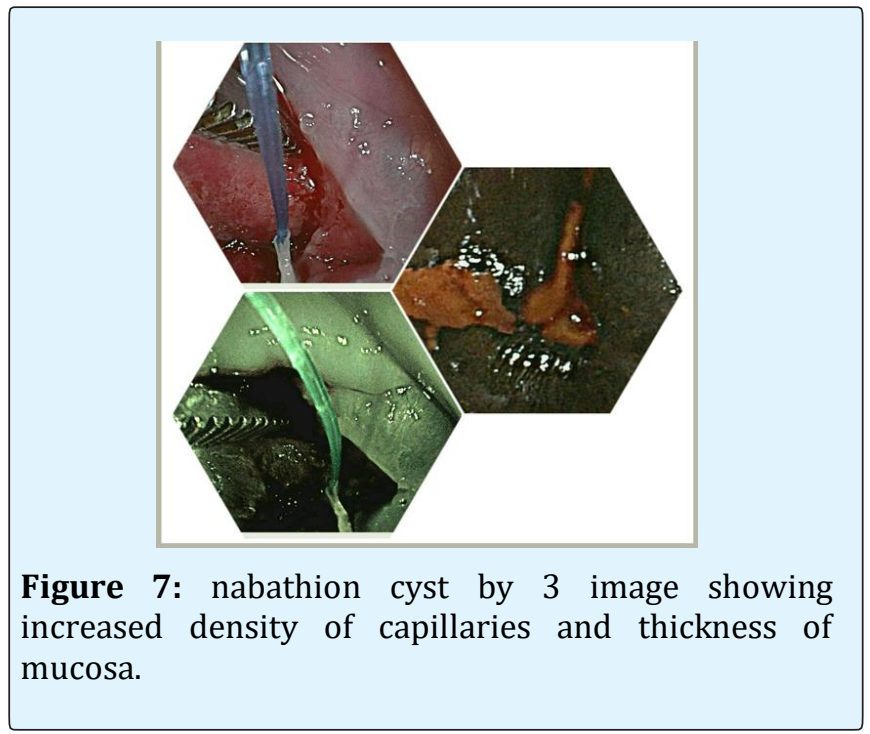

Anter SS. Flexible Endoscopy and Cervical Dysplasia Grade 1 and 2. Ann Adv Biomed Sci 2019, 2(1): 000127.
Area of metapastic cells with evidence from above down area of columnar cells and immature dysplastic cells in different stage figure 8,8 a.

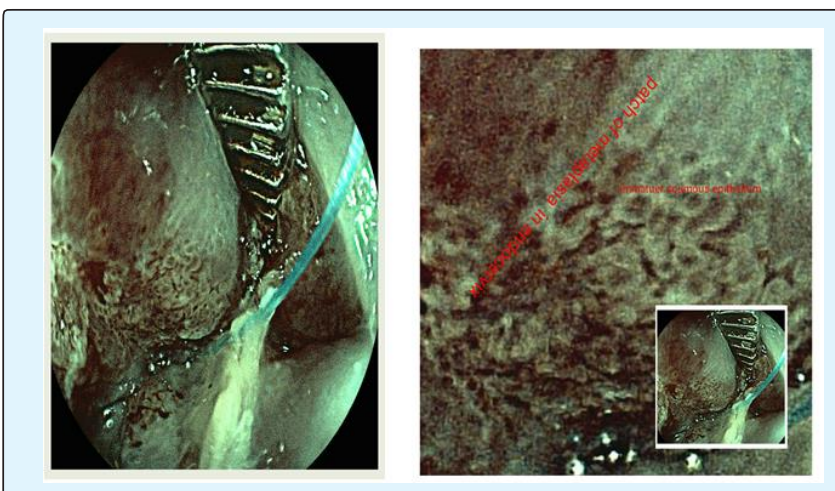

Figure 8: Area of metapastic cells with evidence from above down area of columnar cells and immature dysplastic cells in different stage. 


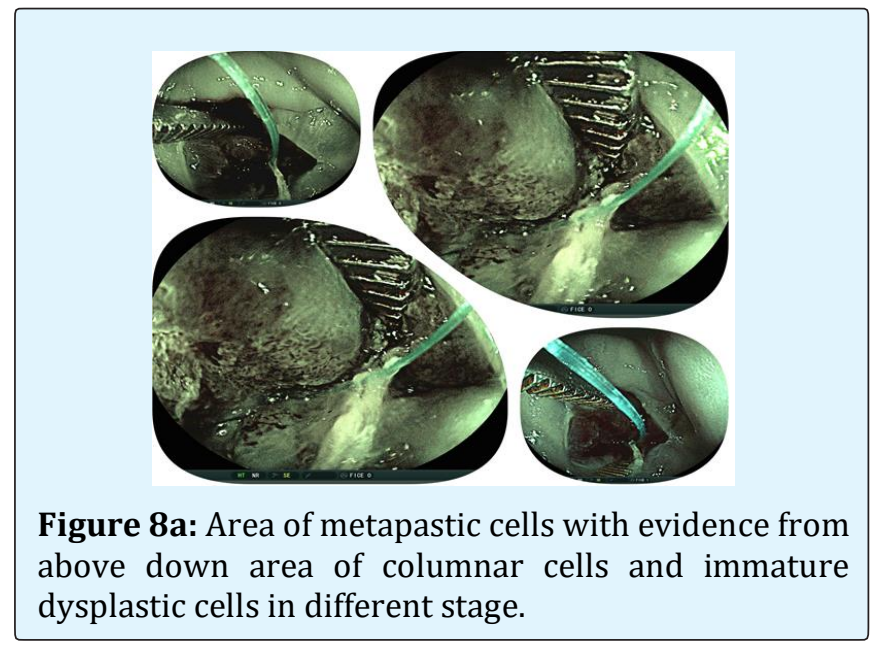

Reserve cells at squamous columnar junction which is cuboid in shape uniform in shape and round nucleoid to by different from dysplastic cells figure 9 it must different from gland occlusion by the first surrounding by halo of acetowhite.

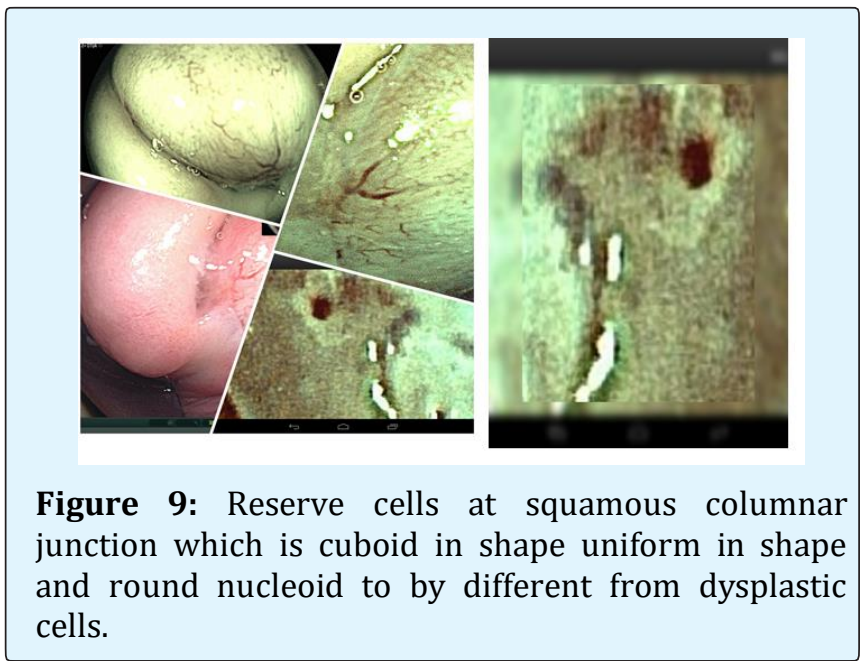

Black point It must different from gland occlusion by the first surrounding by halo of acetowhite Figure 10.

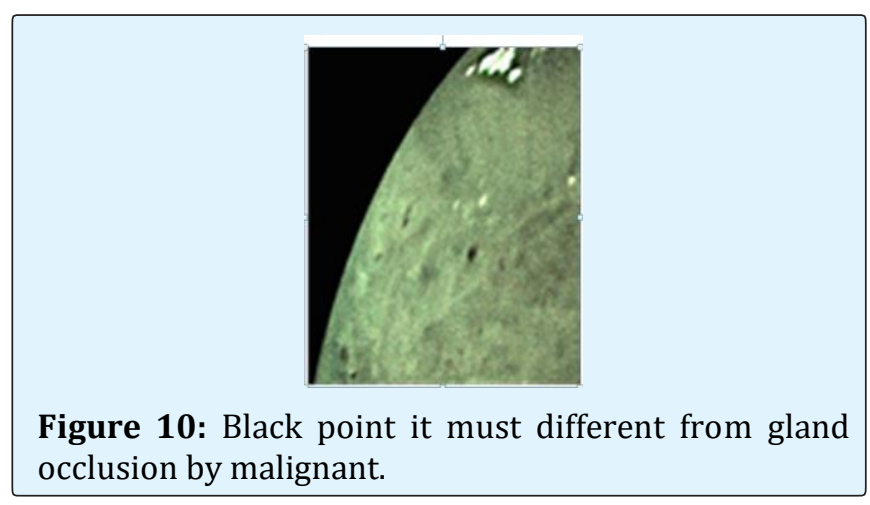

Anter SS. Flexible Endoscopy and Cervical Dysplasia Grade 1 and 2. Ann Adv Biomed Sci 2019, 2(1): 000127.
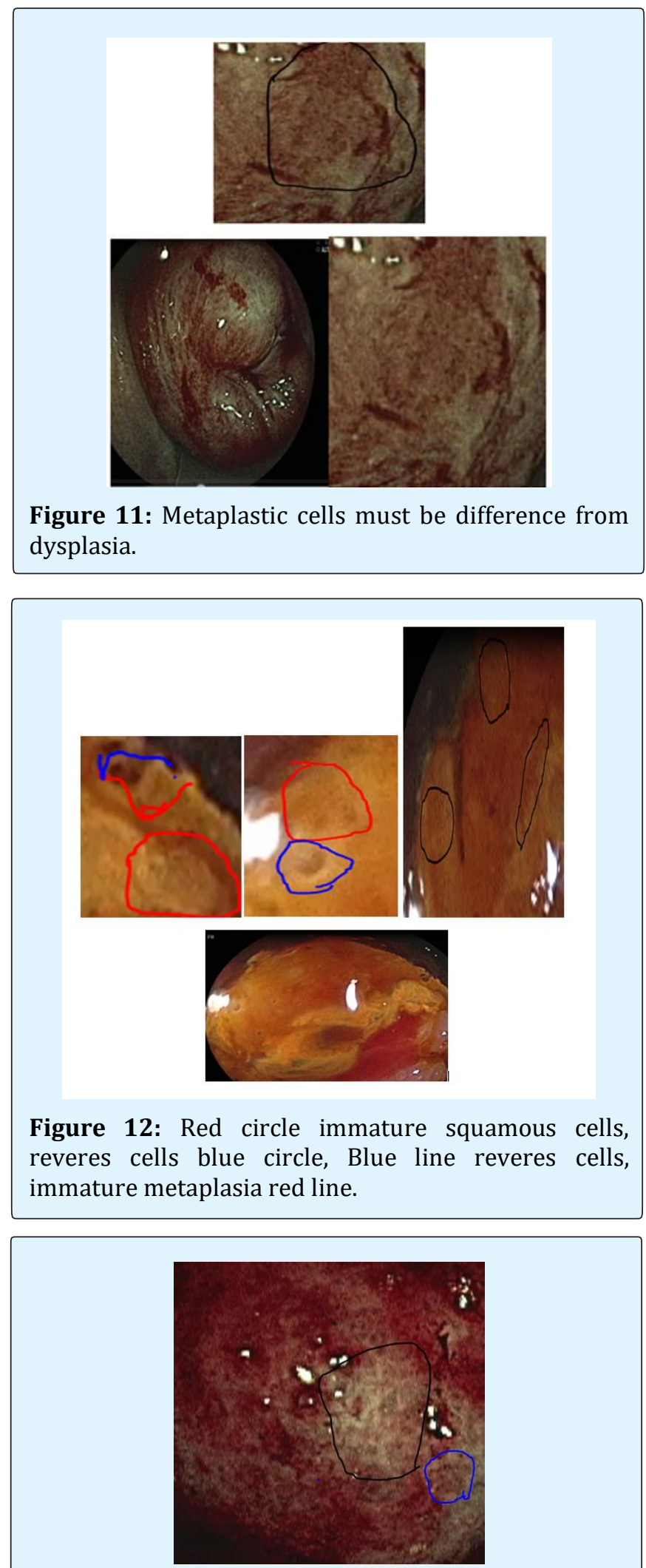

Figure 13: Immature metaplasia black line. 


\section{References}

1. Gono K, Obi T, Yamaguchi M, Ohyama N, Machida H, et al. (2004) Appearance of enhanced tissue features in narrow-band endoscopic imaging. J Biomed Opt 9(3): 568-577.

2. Hirata I, Nakagawa Y, Ohkubo M, Yahagi N, Yao K (2012) Usefulness of magnifying narrow-band imaging endoscopy for the diagnosis of gastric and colorectal lesions. Digestion 85(2): 74-79.

3. Tanaka S, Sano Y (2011) Aim to unify the narrow band imaging (NBI) magnifying classification for colorectal tumors: Current status in Japan from a summary of the consensus symposium in the 79th annual meeting of the Japan gastroenterological endoscopy society. Dig Endosc 23(S1): 131-139.

4. Nishiyama N, Kanenishi K, Mori H, Kobara H, Fujihara S, et al. (2017) Flexible magnifying endoscopy with narrow band imaging for the diagnosis of uterine cervical tumors: a cooperative study among gastrointestinal endoscopists and gynecologists to explore a novel micro-vascular classification system. Oncol Lett 14(1): 355-362.
5. Uchita K, Kanenishi K, Hirano K, Kobara H, Nishiyama $\mathrm{N}$, et al. (2018) Characteristic findings of high-grade cervical intraepithelial neoplasia or more on magnifying endoscopy with narrow band imaging. Int J Clin Oncol 23(4): 707-714.

6. Schiffman M, Wentzensen N (2010) From human papillomavirus to cervical cancer. Obstet Gynecol 116(1): 177-185.

7. Koeneman MM, Kruitwagen RF, Nijman HW, Slangen BF, van Gorp T, et al. (2015) Natural history of highgrade cervical intraepithelial neoplasia: A review of prognostic biomarkers. Expert Rev Mol Diagn 15(4): 527-546.

8. McCredie MRE, Sharples KJ, Paul C, Baranyai J, Medley G, et al. (2008) Natural history of cervical neoplasia and risk of invasive cancer in women with cervical intraepithelial neoplasia 3: A retrospective cohort study. Lancet Oncol 9(5): 425-434.

9. NHS (2004) Colposcopy and programme management: Guidelines for the NHS cervical screening programme. NHSCSP. 